\title{
Ion Temperature Anisotropy Limitation In High Beta Plasmas
}

\author{
Earl E. Scime \\ Paul A. Keiter \\ Matthew M. Balkey \\ Robert F. Boivin \\ John L. Kline \\ See next page for additional authors
}

Follow this and additional works at: https://researchrepository.wvu.edu/faculty_publications

\section{Digital Commons Citation}

Scime, Earl E.; Keiter, Paul A.; Balkey, Matthew M.; Boivin, Robert F.; Kline, John L.; Blackburn, Melanie; and Gary, S. Peter, "Ion Temperature Anisotropy Limitation In High Beta Plasmas" (2000). Faculty Scholarship. 572.

https://researchrepository.wvu.edu/faculty_publications/572 
Authors

Earl E. Scime, Paul A. Keiter, Matthew M. Balkey, Robert F. Boivin, John L. Kline, Melanie Blackburn, and S. Peter Gary 


\title{
Ion temperature anisotropy limitation in high beta plasmas*
}

\author{
Earl E. Scime ${ }^{\dagger}{ }^{\dagger}$ Paul A. Keiter, Matthew M. Balkey, Robert F. Boivin, \\ John L. Kline, and Melanie Blackburn \\ West Virginia University, Morgantown, West Virginia 26506 \\ S. Peter Gary \\ Los Alamos National Laboratory, Los Alamos, New Mexico 87544
}

(Received 5 November 1999; accepted 4 January 2000)

\begin{abstract}
Measurements of parallel and perpendicular ion temperatures in the Large Experiment on Instabilities and Anisotropies (LEIA) space simulation chamber display an inverse correlation between the upper bound on the ion temperature anisotropy and the parallel ion beta ( $\beta$ $\left.=8 \pi n k T / B^{2}\right)$. Fluctuation measurements indicate the presence of low frequency, transverse, electromagnetic waves with wave numbers and frequencies that are consistent with predictions for Alfvén Ion Cyclotron instabilities. These observations are also consistent with in situ spacecraft measurements in the Earth's magnetosheath and with a theoretical/computational model that predicts that such an upper bound on the ion temperature anisotropy is imposed by scattering from enhanced fluctuations due to growth of the Alfvén ion cyclotron instability. (C) 2000 American Institute of Physics. [S1070-664X(00)91405-8]
\end{abstract}

\section{INTRODUCTION}

Historically, the effects of small-scale, collective processes in collisionless plasmas have been represented by analogues of collision-dominated transport coefficients. However, measurements in collisionless space plasmas and hybrid-kinetic simulations suggest that some of these processes can be parameterized with simple expressions of a few variables. In this work, we examine the flow of perpendicular ion thermal energy into parallel ion thermal energy in a high beta, laboratory plasma. In collisional plasmas, such energy flow results from Coulomb scattering. In collisionless plasmas, it is generally believed that small wavelength instabilities can grow at the expense of the energy stored in anisotropic particle distributions. Such instabilities then reduce the anisotropy through velocity space diffusion arising from wave-particle interactions.

The threshold conditions for, and characteristics of, anisotropy driven instabilities have been the subject of numerous experimental, theoretical, and computational investigations. However, the focus of this work is the net effect of a particular class of such instabilities. Given that temperature anisotropies will lead to the excitation of microinstabilities, is it possible to predict the effect on the macroscopic properties of the system? For example, the various published confinement scalings of thermonuclear fusion experiments describe an empirical relationship between the macroscopic properties of the system, e.g., temperature and confinement time, that results from the effects of microinstability driven particle and energy transport. ${ }^{1}$ In the collisionless plasmas of certain regions of space, it appears that the isotropization of ions can be described by a simple expression of the form, ${ }^{2-5}$

*Paper HI2 1 Bull. Am. Phys. Soc. 44, 156 (1999).

${ }^{\dagger}$ Invited speaker.

$$
\frac{T_{i \perp}}{T_{i \|}}-1=\frac{S_{p}}{\beta_{i \|}^{\alpha_{p}}} .
$$

Here $S_{p}$ and $\alpha_{p}$ are dimensionless fitting parameters, $\beta_{i \|}$ $=8 \pi n k T_{i \|} / B_{0}^{2}$, and $T_{i \|}$ and $T_{i \perp}$ are the ion temperatures measured parallel and perpendicular to the local magnetic field, $B_{0}$. When large data sets are examined, Eq. (1) with $\alpha_{p} \approx 0.5$ and $0.5<S_{p}<1$ appears to represent an upper bound on the ion temperature anisotropy for a given value of parallel ion beta, $\beta_{i \|}$ in the terrestrial magnetosheath. The magnetosheath is a region of space near the Earth that consists primarily of shocked solar wind plasma. Because the Earth's bow shock heats the solar wind ions in the direction perpendicular to $B_{0}$, the ion distributions in the magnetosheath are consistently bi-Maxwellian with $T_{\mathrm{i} \perp}>T_{\mathrm{i}||} \cdot{ }^{6}$

Theoretical investigations of the stability of collisionless anisotropic plasmas indicate that two instabilities are likely to grow in the high beta, $\beta \sim 1$, anisotropic, $T_{i \perp}>T_{i \|}$, conditions of the magnetosheath: the mirror mode $\mathrm{e}^{7,8}$ and the $\mathrm{Al}-$ fvén Ion Cyclotron Instability (also known as the anisotropic ion cyclotron instability). ${ }^{9-11}$ The Alfvén Ion Cyclotron (AIC) instability has a real frequency $\omega_{r}$ which satisfies 0 $<\omega_{r}<\Omega_{i}\left(\Omega_{i}\right.$ is the ion cyclotron frequency), whereas $\omega_{r}$ $=0$ for the mirror mode in a homogeneous plasma. The magnetic fluctuations of the ion cyclotron anisotropy mode are directed primarily perpendicular to $B_{0}$. Mirror mode fluctuations are primarily compressive, that is, $\Delta B \| B_{0}$. Electromagnetic fluctuation measurements in the magnetosheath suggest the presence of both mirror and AIC instabilities, with the former typically present when $\beta_{i \|}>1$, and the latter arising when $\beta_{i \|} \leqslant 1$. The question posed earlier can now be recast into a form specific to this anisotropy: "Assuming the existence of mirror and/or AIC instabilities, is it possible to predict the observed beta dependent upper bound on the ion temperature anisotropy ?" If it is possible to make such a 
prediction, then the diagonal elements of the ion pressure tensor in the second moment of the Vlasov equation (the energy equation) can be directly related to each other and the number of free parameters in the ion energy equation reduced. In other words, partial closure of the kinetic equations could be effected even though the mean free path of the particles is large compared to the system size (the limit in which standard Chapman-Enskog asymptotic closure techniques fail).

To relate the ion temperature anisotropy to the plasma beta requires the additional assumption that a plasma instability threshold derived from linear theory corresponds to an observable bound on the anisotropy driving the unstable mode. ${ }^{12}$ In combination with the assumption that waveparticle scattering by enhanced fluctuations from kinetic plasma instabilities constrains the anisotropies that drive the unstable modes, linear theory can be used to predict the upper bound on the ion temperature anisotropy in a high beta collisionless plasma. Recent one-dimensional hybrid simulations of plasmas with large initial ion temperature anisotropies have demonstrated that the initial ion temperature anisotropy is reduced by wave-particle scattering to a level that depends inversely on beta in agreement with the upper bound predicted by linear theory. ${ }^{13}$

The threshold anisotropy for the ion cyclotron anisotropy mode is a function of the ionic constituents of the plasma $^{14}$ and typically has the lower threshold when $\beta_{i \|}$ $=8 \pi n k T_{i \|} / B_{0}^{2}<1$. Since the mirror instability is more likely to arise under conditions of relatively high parallel ion beta, ${ }^{15}$ it is not likely to arise in our experiments and will not be considered further. Linear Vlasov theory calculations indicate that the threshold condition for onset of the ion cyclotron anisotropy instability for a fixed value of the dimensionless maximum growth rate $\gamma / \Omega_{i}$ in an electron/ion plasma can also be described by a relationship of the form of Eq. (1). ${ }^{16}$ In this case, $S_{p}$ is of the order of unity (determined by the choice of maximum growth rate) and $\alpha_{p}$ is relatively independent of $\gamma / \Omega_{i}$ (typically $\alpha_{p} \approx 0.4$ ). The threshold conditions for excitation of AIC instabilities have been studied before in laboratory experiments. ${ }^{17-19}$ While those experiments did report AIC instabilities for plasma parameters exceeding the AIC instability threshold, ${ }^{10}$ they did not report evidence of an upper bound on the ion temperature anisotropy. It is worth noting that those experiments were limited to indirect measurements of the ion temperature anisotropy and were transient, i.e., pulsed, experiments.

As mentioned earlier, in situ measurements in the magnetosheath have suggested that there is an upper bound on the ion temperature anisotropy in the terrestrial magnetosheath that also satisfies Eq. (1) with $\alpha_{p} \approx 0.5$. Electromagnetic fluctuation measurements obtained at the same time show evidence of AIC instabilities, ${ }^{4}$ mirror modes waves, ${ }^{4}$ or unidentified broadband, low frequency, electromagnetic waves. ${ }^{20}$ In addition, magnetosheath ion distributions are often observed to exhibit more than one type of nonMaxwellian feature, thus scattering may be due to more than one microinstability.

In a carefully controlled, fully diagnosed, high beta plasma, we have carried out the first laboratory demonstra-

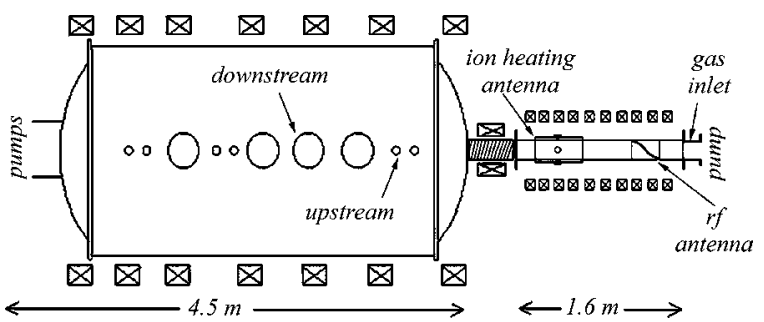

FIG. 1. The Large Experiment on Instabilities and Anisotropies (LEIA) connected to the helicon plasma source (right). The two locations at which LIF measurements were performed in LEIA are indicated.

tion of the existence of an upper bound on the ion temperature anisotropy that scales inversely with the ion beta. Initial results from these experiments have been reported elsewhere. ${ }^{21}$ Here we provide detailed descriptions of the experimental apparatus, the ion distribution functions, the anisotropy vs parallel ion beta measurements, and the electromagnetic wave measurements. The experimental results confirm the predictions of collisionless theory and simulations, as well as validating results of space plasma observations carried out with less comprehensive diagnostics and under natural, rather than controlled conditions.

\section{EXPERIMENTAL APPARATUS}

The experiments were performed in the Large Experiment on Anisotropies and Instabilities (LEIA). LEIA (Fig. 1) consists of a steady state, high density, helicon plasma source (HELIX) coupled to a large (4.4 m long, $1.8 \mathrm{~m}$ inner diameter) vacuum chamber. LEIA is specifically designed to study space-relevant instabilities driven by particle distributions in high $\beta\left(\beta \gg m_{\mathrm{e}} / m_{i}\right)$ plasmas. It is important to note that because LEIA plasmas are not in magnetohydrodynamic (MHD) equilibrium, high $\beta$ plasmas can be generated without creating a significant diamagnetic cavity in the plasma. The change in on-axis axial magnetic field as a function of electron $\beta$ is shown in Fig. 2. Essentially, the combination of no axial confinement and marginal collisionality is sufficient

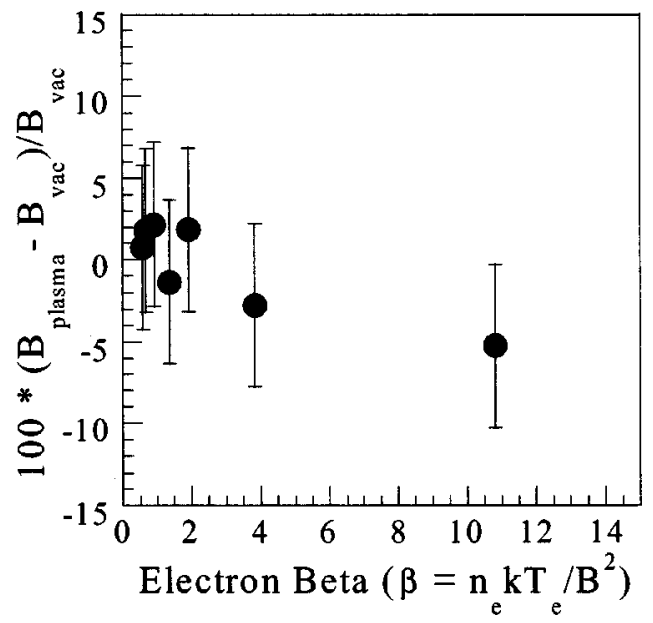

FIG. 2. The change in the vacuum axial magnetic field due to the presence of the plasma as a function of electron $\beta$. 




FIG. 3. Plasma density vs radius for typical LEIA parameters used in these experiments.

to prevent MHD equilibrium from being established. Therefore, the axial magnetic field is only slightly reduced for $\beta$ greater than unity.

Typical LEIA argon plasma parameters for these experiments were $n \leqslant 10^{12} \mathrm{~cm}^{-3}, B \approx 17-70 \mathrm{G}, T_{e} \approx 4-10 \mathrm{eV}, T_{i}$ $\approx 0.1-1.0 \mathrm{eV}, \beta_{e \|} \leqslant 0.2$ and $\beta_{i \|} \leqslant 0.02$. A representative density profile is shown in Fig. 3. For these parameters, the radial density profile is clearly hollow. The density and temperature profiles were measured with a RF compensated Langmuir probe. ${ }^{22}$ Langmuir probe measurements made at different axial positions indicate that the plasma density decreases roughly a factor of 2 along the length of LEIA. A radial profile of normalized electrostatic fluctuation amplitude measured with an uncompensated Langmuir probe for a LEIA magnetic field of $18 \mathrm{G}$ is shown in Fig. 4. The Langmuir probe was driven into ion saturation with a $-96 \mathrm{~V}$ bias and fluctuations in the ion saturation current normalized to the overall ion saturation current were used for the data shown in Fig. 4. An uncompensated Langmuir probe was used because the inductive chokes in a standard RF compensated probe ${ }^{22}$ also pick up electromagnetic fluctuations. Note that the overall normalized electrostatic fluctuation amplitude is less than $1 \%$ for the region examined.

Because of the differential pumping scheme, the neutral pressure decreases from 1.4 mTorr in the source where the gas is injected, to 0.2 mTorr in LEIA. At these ion tempera-

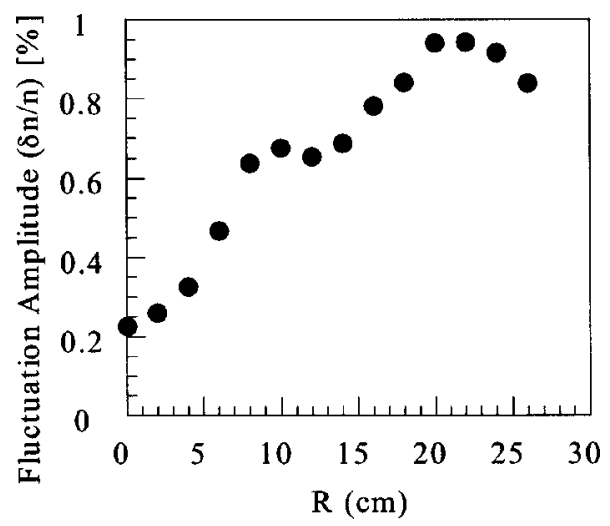

FIG. 4. Normalized electrostatic fluctuation amplitude vs radius.

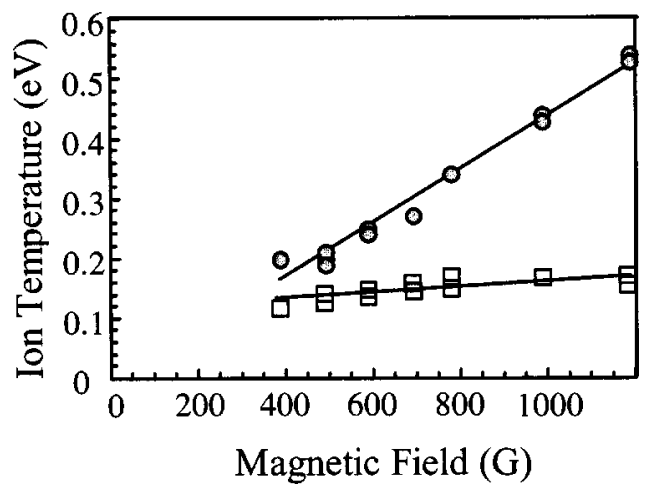

FIG. 5. Perpendicular ion temperature (filled circles) and parallel ion temperature (open squares) vs magnetic field strength in the helicon source.

tures and magnetic fields, the plasma radius is eight times the ion gyroradius. The plasma is marginally collisional as $\lambda_{\mathrm{mfp}} / \rho \approx 1$ for the ion-ion collisions $\left(\lambda_{\mathrm{mfp}}\right.$ is the mean free path and $\rho$ is the ion gyroradius). Based on the edge neutral pressure, $\lambda_{\mathrm{mfp}} / \rho \approx 1$ for ion-neutral collisions. ${ }^{23}$ This is an overestimate for ion-neutral collisions as the neutral pumping effect in helicon plasmas should lead to a significantly reduced neutral pressure on axis in LEIA. ${ }^{24}$ The ion-ion collision frequency ranges from 5 to 10 times the ion gyrofrequency.

The parallel and perpendicular ion temperatures in both the helicon source and in LEIA were determined by laser induced fluorescence (LIF). ${ }^{25,26}$ In the helicon source, the perpendicular ion temperature is a strong function of the source magnetic field strength, while the parallel ion temperature is relatively independent of the source magnetic field (see Fig. 5). Because such large intrinsic ion temperature anisotropies in the source were unanticipated, the helicon source was designed with an auxiliary ion heating system. ${ }^{27}$ However, the auxiliary ion heating system was only used in these experiments to suppress the low frequency electromagnetic waves believed to be associated with the ion temperature anisotropy relaxation. The low frequency wave suppression process will be discussed in more detail when the wave measurements are reviewed.

In LEIA, the ion velocity distribution is bi-Maxwellian with $T_{i \perp}>T_{i \|}$ (Fig. 6). The data shown in Fig. 6 are from measurements of the ion velocity space distribution at 36 different angles with respect to the axial magnetic field. The measurements were obtained with an in situ tomographic LIF probe ${ }^{28}$ located at the downstream port (see Fig. 1) and then processed with a filtered back projection algorithm to obtain the two-dimensional velocity space distribution function. ${ }^{29,30}$ The perpendicular and parallel ion temperatures are also measured at the upstream port (Fig. 1) with a fixed set of injection and collection optics. As in the helicon source, there is an intrinsic ion temperature anisotropy in LEIA. For typical plasma parameters, the ion temperature anisotropy vs magnetic field strength in LEIA for a fixed value of helicon source magnetic field is shown in Fig. 7. If the plasma expanded adiabatically into the weaker magnetic field of LEIA, magnetic moment conservation would increase the parallel ion energy at the expense of the perpen- 




FIG. 6. Contours of constant phase space density as a function of parallel and perpendicular ion velocity in LEIA. Note that the perpendicular ion temperature is greater than the parallel ion temperature.

dicular ion temperature. However, the perpendicular ion temperature in LEIA is often observed to be greater than the perpendicular ion temperature in the helicon source. Thus, in addition to the mechanism heating the ions in the helicon source, there must be another process that heats the ions as the plasma expands into LEIA.

The strong correlation between LEIA perpendicular ion temperature and flow velocity from the source into LEIA (see Fig. 8) suggests that parallel velocity shear may play an important role in driving the ion temperature anisotropy in LEIA. ${ }^{31}$ The data of Fig. 8 were obtained by varying the neutral gas pressure in the helicon source while measuring the perpendicular ion temperature in LEIA at the upstream port and the parallel flow velocity in the helicon source. Measurements of the total ion energy at both positions indicate that the total ion energy is conserved, therefore some mechanism is converting parallel ion flow into perpendicular ion temperature. Assuming that the flow goes to zero at the walls, sufficient shear in the parallel flow may exist to excite parallel velocity shear driven instabilities. ${ }^{31}$ Regardless of how the ions get heated in the perpendicular direction, the experiments described here concern the evolution of ion ve-

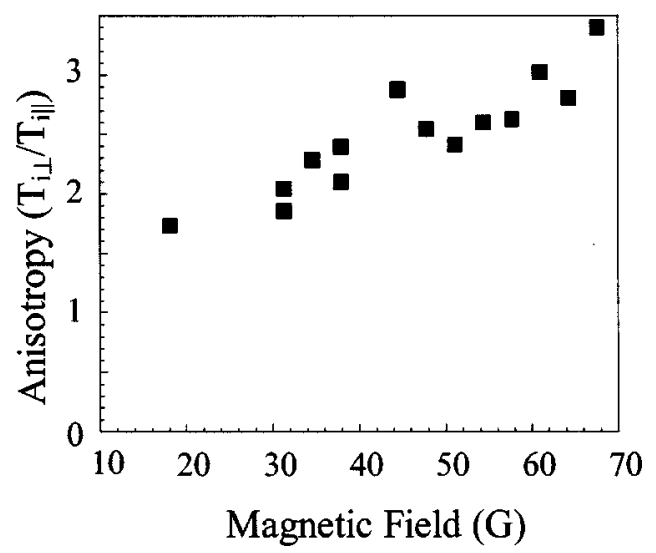

FIG. 7. Ion temperature anisotropy in LEIA vs LEIA magnetic field strength.



FIG. 8. Perpendicular ion temperature in LEIA vs drift velocity of the ions along the field in the helicon source for LEIA magnetic fields of $35 \mathrm{G}$ (open squares) and $65 \mathrm{G}$ (open circles).

locity space distribution after such heating. We ask, does the resultant ion temperature anisotropy depend on the ion $\beta$ and are low frequency electromagnetic waves excited when the ion temperature anisotropy is large?

\section{ION TEMPERATURE ANISOTROPY MEASUREMENTS}

Ion temperature anisotropy measurements, $T_{i \perp} / T_{i \|}$ vs $\beta_{i \|}$ at the upstream position in LEIA are shown in Fig. 9. The data shown here were all obtained for the same neutral pressure and source if power. Different source magnetic fields and LEIA magnetic fields were used to vary the anisotropy and $\beta_{i \|}$. Each measurement of ion temperature anisotropy and $\beta_{i \|}$ is based on multiple ion temperature mea-

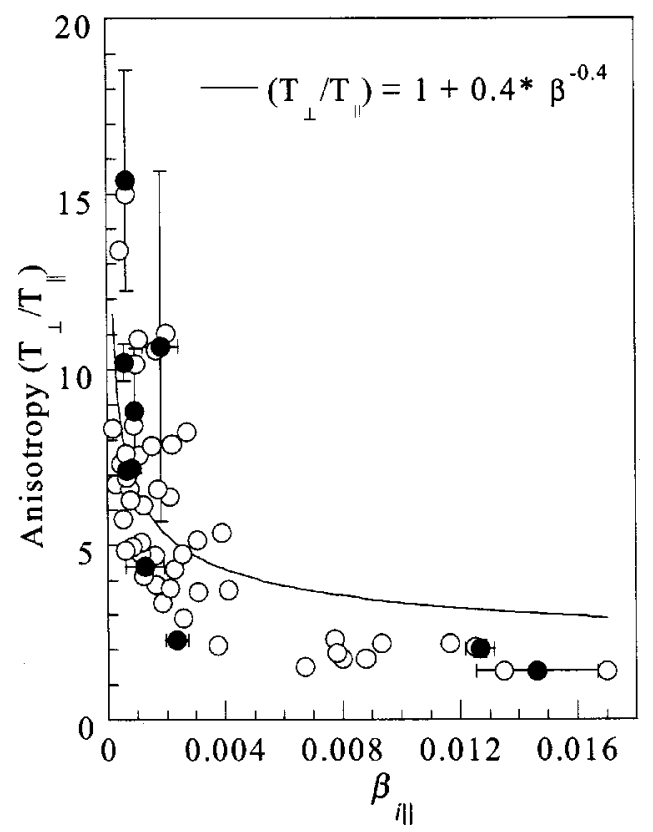

FIG. 9. The ion temperature anisotropy, $A=T_{i \perp} / T_{i \|}$, vs $\beta_{i \|}$ (open circles) measured at the upstream position. These data were obtained over a wide range of operating magnetic fields but at fixed rf power and neutral pressure. Also shown are averaged values of anisotropy and $\beta_{i \|}$ for similar operating conditions (solid circles) investigated on different days with standard deviation error bars. 
surements at a particular set of operational parameters. Average ion temperature anisotropy and $\beta_{i \|}$ values from experiments on different days but at similar operational parameters are shown as solid circles in Fig. 9. The associated error bars are the standard deviations in the measurements at those operating conditions. Variations in the parallel ion temperature measurements due to poor signal to noise ratio for parallel measurements are responsible for the bulk of the variance in the measurements. Also shown in Fig. 9 is a power law fit to the measurements. The power law exponent obtained from the fit is consistent with both the magnetosheath observations and the computational results.

The most significant difference between these experiments and the magnetosheath observations is the collisionality of the laboratory plasma. The linear Vlasov model used to describe the growth and characteristics of both the mirror and AIC waves also assumes a collisionless plasma. Although it is not possible to eliminate collisional effects in these experiments, it is possible to examine the scaling of ion temperature anisotropy with $\beta_{i \|}$ in LEIA within the constraint of constant ion-ion and ion-neutral collision frequencies (ion-electron collisions have a negligible effect on the ion temperature anisotropy). For a bi-Maxwellian ion distribution, the rates of change for the perpendicular and parallel ion temperatures are $^{32}$

$$
\frac{d T_{\perp}}{d t}=-\frac{1}{2} \frac{d T_{\|}}{d t}=-\nu\left(T_{\perp}-T_{\|}\right),
$$

where

$$
\nu \approx \frac{2 \sqrt{\pi} e^{4} n}{\sqrt{m_{i}}\left(k T_{\|}\right)^{3 / 2} A^{2}}\left[-3+(A+3) \frac{\tan ^{-1}(\sqrt{A})}{\sqrt{A}}\right]
$$

and $A=T_{i \perp} / T_{\|}$. For typical LEIA parameters, the collisional isotropization frequency can be approximated by

$$
\nu \approx \frac{4 \sqrt{\pi} e^{4} n}{\sqrt{m_{i}}\left(k T_{\|}\right)^{3 / 2} A^{0.9}} .
$$

Rewriting $\beta_{i \|}$ in terms of Eq. (4),

$$
\beta_{i \|} \approx \frac{\nu\left(k T_{\|}\right)^{5 / 2} A^{0.9}}{4 \sqrt{\pi / m_{i}} e^{4} B^{2}} .
$$

Thus, by taking advantage the independent control of magnetic field, ion temperature, and density afforded by the helicon source and space chamber combination, a wide range of $\beta_{i \|}$ is accessible for a fixed value of isotropization frequency. Figure 10(a) shows a subset of the data shown in Fig. 9. For these data, the isotropization frequency described by Eq. (3) varies by about $10 \%$. Note that $\beta_{i \|}$ spans more than order of magnitude and the temperature anisotropy changes by a factor of 3.

If we assume that some source of ion heating exists and that ion-ion collisions are responsible for all of the ion thermalization, we can estimate the $\beta_{i \|}$ scaling that would result. Adding an energy source term, $H$, to Eq. (3),

$$
\frac{d T_{\perp}}{d t}=H-\nu\left(k T_{\perp}-k T_{\|}\right) .
$$
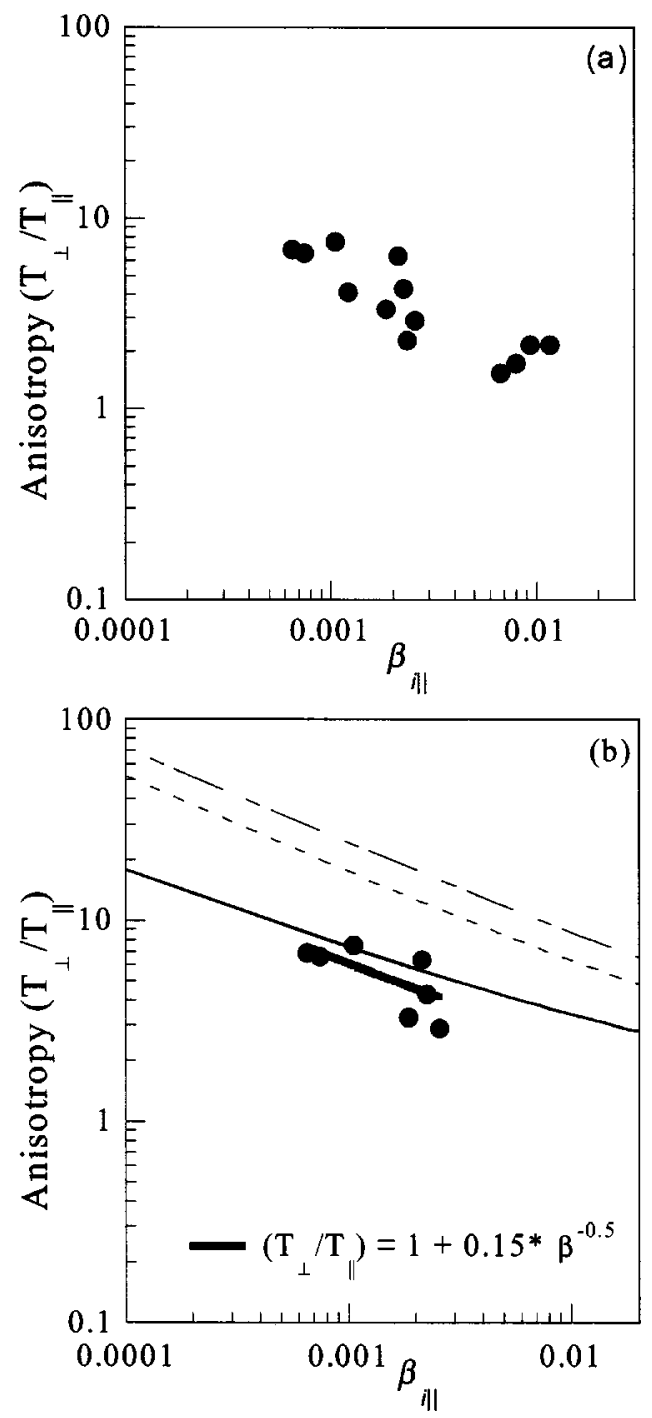

FIG. 10. (a) A subset of the ion temperature anisotropy, $A=T_{i \perp} / T_{i \|}$, vs $\beta_{i \|}$ data of Fig. 9 selected so that the isotropization rate, Eq. (2), varies by $10 \%$. (b) The same data as in part (a) with the additional constraint of fixed ion-neutral collision frequency (10\% variation). Also shown are the fits to Eq. (1) for the laboratory measurements (heavy solid line), the magnetospheric observations of Phan et al. (short dashes) (Ref. 3) the magnetospheric observations of Anderson et al. (long dashes) (Ref. 2) and the linear Vlasov calculations with $\gamma=10^{-4} \Omega_{p}$ (thin solid line) (Ref. 16).

In steady state, which is true for LEIA, and using the approximate form for the collision frequency of Eq. (4), Eq. (6) becomes

$$
\frac{T_{\perp}}{T_{\|}}=1+\frac{H \sqrt{m_{i}}\left(k T_{\|}\right)^{1 / 2} A^{0.9}}{4 \sqrt{\pi} e^{4} n} .
$$

Using the measured scalings of ion temperature anisotropy vs magnetic field (Fig. 7), plasma density and magnetic field vs $\beta_{i \|}$, and assuming the source function is independent of LEIA parameters, Eq. (7) can be rewritten as a function of only $\beta_{i \|}$,

$$
\frac{T_{\perp}}{T_{\|}}=1+\frac{H\left(0.03+0.37 \beta^{0.4}\right)}{C \beta} \frac{(1+108 \beta)}{\sqrt{(1+225 \beta)}},
$$


where $C$ is a constant. Over the range $0.001<\beta_{i \|}<0.01$, according to Eq. (8) the ion temperature anisotropy should scale roughly as $\beta_{i \|}^{-0.3}$. Thus, based on how densities and temperatures in the LEIA system scale with $\beta_{i \|}$, the observed decrease in ion temperature anisotropy is steeper than would be expected for ion-ion collisions alone. Because the anisotropy itself plays a role in Eq. (7), the inverse $\beta_{i \|}$ scaling still seen in the data shown in Fig. 10(a) offers the strongest evidence that ion-ion collisions alone cannot explain the observations.

In addition to ion-ion collisions, ion-neutral collisions also reduce temperature anisotropy through velocity space diffusion. For cold argon neutrals and argon ions within the energy range $0.1-1.0 \mathrm{eV}$, the momentum transfer cross section remains roughly constant at about $1 \times 10^{-14} \mathrm{~cm}^{2} .{ }^{33}$ Thus, the ion-neutral collision frequency is proportional to $\sqrt{T_{i}}$, where $T_{i}^{2}=\left(2 T_{i \perp}^{2}+T_{i \|}^{2}\right) / 3$. Figure 10(b) shows a subset of the data shown in Fig. 10(a). For these data, both the isotropization and ion-neutral collision frequencies vary by about $10 \%$. Although the range of $\beta_{i \|}$ is reduced, the inverse correlation of ion temperature anisotropy with $\beta_{i \|}$ apparent in Figure 9 is still apparent. A fit of Eq. (1) to the data yields $S_{p}=0.15$ and $\alpha_{p}=0.5$. Also shown in Fig. 10 are the fits to the upper bound for the magnetosheath observations of Anderson et al., $\left(S_{p}=0.85, \alpha_{p}=0.48\right),{ }^{2}$ the magnetosheath observations of Phan et al., $\left(S_{p}=0.63, \alpha_{p}=0.50\right),{ }^{3}$ and the linear Vlasov theory curve for the onset of the ion cyclotron anisotropy instability at a maximum growth rate of $\gamma / \Omega_{i}$ $=10^{-4} \cdot{ }^{6}$

\section{ELECTROMAGNETIC WAVE MEASUREMENTS}

Electromagnetic fluctuations in LEIA were measured with array of three-axis magnetic sense coils. ${ }^{28}$ As can be seen in Fig. 11, the power spectra of transverse, $B_{r}$, electromagnetic fluctuations in LEIA during these experiments contain a number of distinct features. Scanning the helicon source magnetic field strength, as shown in Figs. 11(c)11(e), clearly associates spectral features in the $5-20 \mathrm{kHz}$ range with helicon source phenomena. During a scan of LEIA magnetic field strength, the roughly $6 \mathrm{kHz}$, narrowband feature remained unchanged [Figures 11(a)-11(c)]. The presence of the narrowband $6 \mathrm{kHz}$ feature is strongly correlated with the parallel flow shown in Fig. 8. However, the amplitude of the broadband, lower frequency activity around 1-2 kHz, shows a distinct increase with decreasing LEIA magnetic field strength. The total spectral power in the range $\Omega_{i} / 2-\Omega_{i}$, normalized to $\Omega_{i} / 2$, vs parallel ion beta for a LEIA magnetic field strength scan is shown in Fig. 12. The monotonic increase of the fluctuating magnetic field energy with $\beta_{i \|}$ here is consistent with the same trend in the simulation results of Ref. 13. However, the experiment yields a much more rapid increase of this energy than the $|\delta B|^{2} / B_{0}^{2} \sim \beta_{i \|}$ scaling of the computations. The ion cyclotron frequency identified in Fig. 11(c) is based on the magnetic field strength at the magnetic probe. Measurements of other field components indicate that $\widetilde{B}_{r} \gg \widetilde{B}_{z}$, so that the low frequency waves are transverse. ${ }^{28}$ These waves are primarily electromagnetic at high $\beta_{i \|}$ as measurements of the electro-

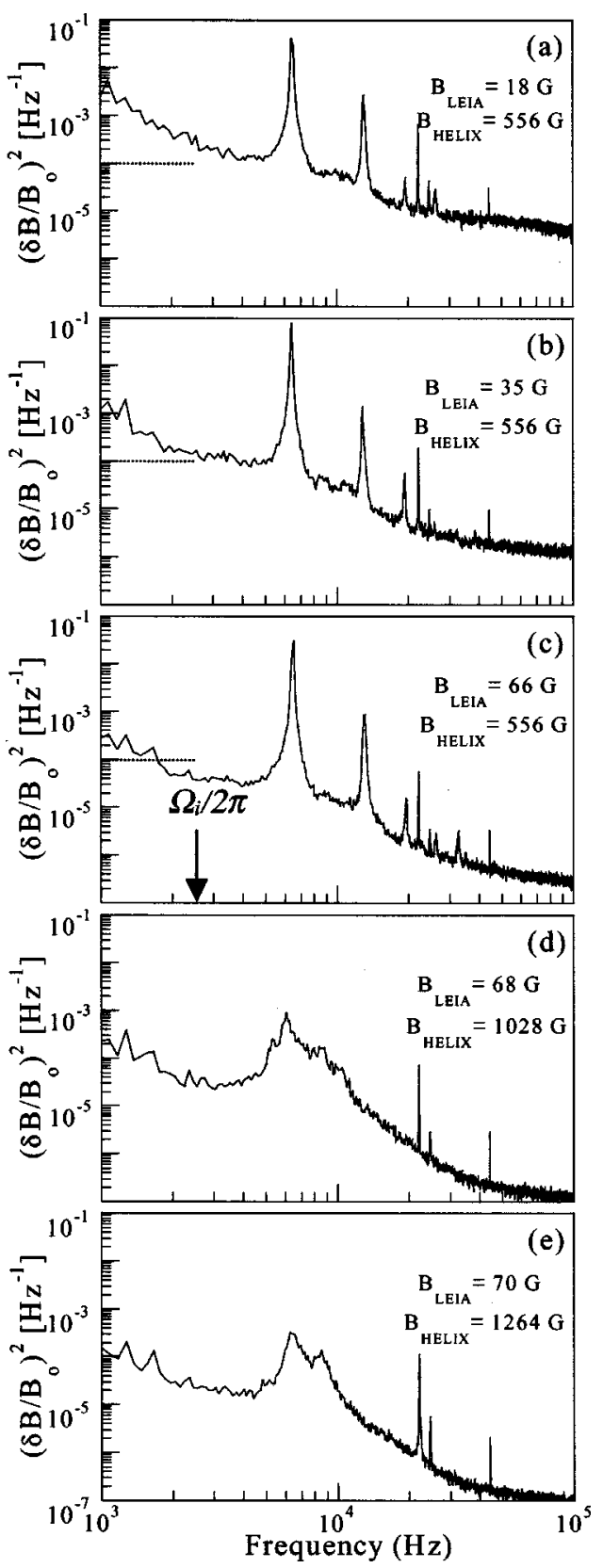

FIG. 11. Power spectra of electromagnetic wave activity in LEIA for different magnetic field configurations (a) $B_{\text {LEIA }}=18 \mathrm{G}, B_{\text {HELIX }}=556 \mathrm{G}$. (b) $B_{\text {LEIA }}=35 \mathrm{G}, B_{\text {HELIX }}=556 \mathrm{G}$. (c) $B_{\text {LEIA }}=66 \mathrm{G}, B_{\text {HELIX }}=556 \mathrm{G}$. (d) $B_{\text {LEIA }}=68 \mathrm{G}, B_{\text {HELIX }}=1028 \mathrm{G}$. (e) $B_{\text {LEIA }}=70 \mathrm{G}, B_{\text {HELIX }}=1264 \mathrm{G}$. The ion cyclotron frequency for a LEIA field of $66 \mathrm{G}$ is shown in (c). The horizontal dashed line is to highlight the change in amplitude of the low frequency waves.

static fluctuation spectrum for the same parameters show a decrease of low frequency electrostatic wave activity with decreasing LEIA magnetic field (see Fig. 13). Thus, these fluctuations appear to be Alfvén ion cyclotron waves, which are transverse, right circularly polarized waves at frequencies below the ion cyclotron frequency.

A typical normalized parallel wave number, $k_{\mathrm{z}}$, spectrum (determined with standard two-point techniques ${ }^{34}$ from magnetic sense coil signals) for high LEIA magnetic field strengths and large levels of ion temperature anisotropy is shown in Fig. 14. The higher LEIA field strengths increase 


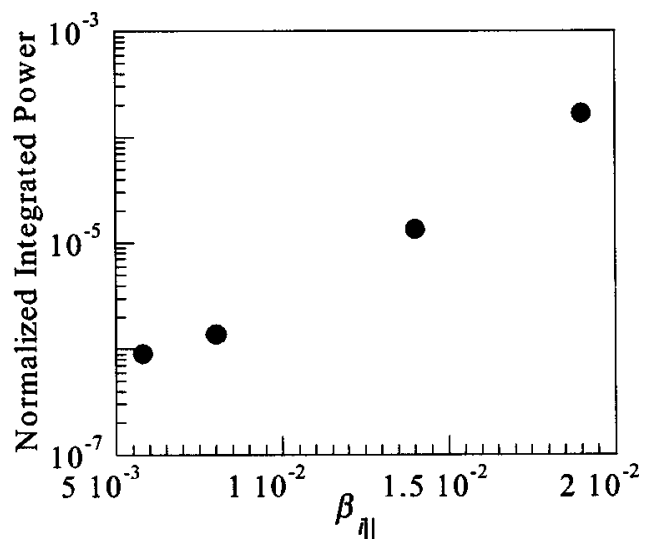

FIG. 12. Magnetic field power spectrum integrated from $\Omega_{i} / 2$ to $1 \Omega_{i}$ and then normalized by $\Omega_{i} / 2$ vs parallel ion beta.

the ion cyclotron frequency, thereby increasing the sensitivity of the sense coil. The high anisotropies yield larger wave amplitudes. For comparison with theory, the wave number spectrum is normalized to $\omega_{p} / c$, where $\omega_{p}$ is the ion plasma frequency. The experimental measurements are in good agreement with theoretical calculations of maximum growth rates and wave numbers for AIC waves that predict a broad parallel wave number spectrum peaked between 0.5 and $2 .{ }^{10}$

\section{CORRELATION OF LOW FREQUENCY WAVE ACTIVITY AND ION ISOTROPIZATION}

To determine if ion temperature anisotropy relaxation can be directly correlated with subcyclotronic electromagnetic wave activity, the ion velocity space distribution was measured at two different axial locations (shown in Fig. 1) for instances when low frequency waves were present and for instances when they were absent. Again, collisional effects could not be eliminated from the experiment, only held constant. The neutral pressure, helicon source magnetic field, and LEIA magnetic field were held constant and the rf power and auxiliary ion heating power manipulated to produce the same upstream plasma density, roughly the same total ion temperature, and different initial ion temperature anisotropies. As mentioned previously, the auxiliary ion heating system ${ }^{27}$ suppresses the low frequency electromagnetic wave activity in LEIA (Fig. 15). Because the ion heating system



FIG. 13. Power spectra of electrostatic fluctuations for two different LEIA magnetic fields. In contrast to the electromagnetic measurements, the low frequency electrostatic wave amplitude decreases with decreasing LEIA magnetic field.



FIG. 14. Measured parallel wave number, $k_{\mathrm{z}}$, spectrum (gray) and heavily smoothed curve (solid line) for ion temperature anisotropy of 8.3 and LEIA magnetic field of $70 \mathrm{G}$.

generates a large, low frequency, $f \sim 30 \mathrm{kHz}$, transverse magnetic perturbation in the helicon source, we hypothesize that this perturbation extends into LEIA where it continues to dominate the magnetic field fluctuations and prevents the growth of the AIC instability. However, because the ion cyclotron frequency is much less than $30 \mathrm{kHz}$ in LEIA, there is little interaction between the $30 \mathrm{kHz}$ magnetic perturbation and the ion velocity space distribution in LEIA.

With similar ion-ion and ion-neutral collision rates, the relative change in ion temperature anisotropy from the upstream to downstream location should be similar if collisions are the only significant isotropizing mechanism in LEIA. For the case with low frequency wave activity, the ion temperature anisotropy decreased from 19 upstream to 2 downstream, i.e., a $90 \%$ decrease. For the case without low frequency wave activity, the anisotropy decreased from 17 upstream to 3 downstream, i.e., an $80 \%$ decrease. The waveparticle interactions provide, at most, a modest amount of ion isotropization in these experiments. However, with low frequency waves present, the downstream distribution is significantly more isotropic and the overall anisotropy reduction is larger.



FIG. 15. Electromagnetic wave power spectra without ion heating in helicon source (solid line) and with ion heating in helicon source (dashed line). The low frequency wave activity is suppressed when the ion heating system is on. 


\section{DISCUSSION}

This work demonstrates that Eq. (1) with $\alpha_{p} \approx 0.5$ constitutes an observable constraint on $T_{i \perp} / T_{i \|}-1$ and that the observed subcyclotron magnetic field fluctuations are correlated with isotropization of the ions. The observed fluctuations are electromagnetic, transverse, and the measured wavenumbers are consistent with predictions for ion cyclotron anisotropy waves. These results strongly suggest that the ion cyclotron anisotropy instability is indeed the process that imposes this bound in both magnetosheath and high $\beta_{i \|}$ laboratory plasmas.

The fact that the ion-ion collision frequency exceeds the ion cyclotron frequency in these experiments raises the question of the applicability of collisionless, linear Vlasov theory to explain the experimental measurements. Whether or not instabilities driven by ion temperature anisotropies in marginally collisional plasmas can be described by linear Vlasov theory is a subject best left for more thorough theoretical analyses. However, similar instabilities have been observed in other marginally collisional experiments and later theoretical analysis indicated that ion-ion collisions changed the thresholds for those instabilities but did not completely suppress their growth. ${ }^{35,36}$

The LEIA plasmas clearly exhibit strong ion temperature anisotropy and low frequency electromagnetic wave activity appears to be correlated with the relaxation of the ion temperature anisotropy. The collisional portion of the anisotropy relaxation most likely scales with the relevant collision frequencies. However, the wave-particle interaction does not necessarily proceed at a rate governed by the ion cyclotron frequency, nor may such interactions require complete gyroorbits to add to the velocity space diffusion of the ions. Regardless of the correct theoretical description of the low frequency, transverse, electromagnetic waves, sufficiently large amplitude waves can result in significant pitch angle scattering of the ions. The pitch angle diffusion coefficient for ion cyclotron turbulence scales as $\left(\Omega_{i}^{2} / v\right) \cdot\left(\delta B / B_{0}\right)^{2}{ }^{37}$ As shown in Fig. 12, the wave power grows exponentially with increasing $\beta_{i \|}$. Therefore, although the plasma is marginally collisional, the pitch angle scattering rate will be a strong function of $\beta_{i \|}$ and such scattering may be responsible for the increased ion isotropization observed when the low frequency waves are observed.

Finally, these results support the conclusion that aspects of energy flow in high beta plasmas can be parameterized in terms of lower order moments of the system, thus providing an alternative to complicated closure methods in theoretical models of systems not typically amenable to simple closure techniques. This idea of using constraints on anisotropies imposed by short wavelength instabilities instead of using analogs of collision-dominated transport coefficients has been suggested in other contexts. For example, the Manheimer and Boris limitation on field-aligned currents ${ }^{12}$ may prove more useful than the various approaches to anomalous resistivity, ${ }^{38}$ and the heat flux constraint imposed by heat flux instabilities may have greater application to collisionless plasmas than the Spitzer-Harm thermal conductivity derived from collision-dominated theory. ${ }^{39}$

\section{ACKNOWLEDGMENTS}

This WVU portion of this work was supported by the National Science Foundation under Grant No. ATM9616467, the U.S. Department of Energy under Grant No. DE-FG02-97ER54420, and NASA under the SEAP program. The Los Alamos portion of this work was conducted under the auspices of the U.S. Department of Energy. We thank Carl Weber, Doug Mathess, and Tom Milam for their efforts in constructing the LEIA facility, our colleagues at MIT, the Max Planck Institute in Garching, the UW-Madison, and LANL for generous donations of the equipment used to construct LEIA, Roger McWilliams and Mike Zintl for the FORTRAN version of the filtered back projection algorithm, and Gabriel Tapia for his programming efforts.

${ }^{1}$ S. D. Scott et al., in Proceedings of the Fourteenth International Atomic Energy Agency Conference on Plasma Physics and Controlled Nuclear Fusion Research (International Atomic Energy Agency, Vienna, 1993), Vol. 3, p. 427.

${ }^{2}$ B. J. Anderson and S. A. Fuselier, J. Geophys. Res. 98, 1461 (1993).

${ }^{3}$ T.-D. Phan, G. Paschmann, W. Baumjohann, and N. Sckopke, J. Geophys. Res. 95, 1015 (1994).

${ }^{4}$ B. J. Anderson, S. A. Fuselier, S. P. Gary, and R. E. Denton, J. Geophys. Res. 99, 5877 (1994).

${ }^{5}$ L. C. Tan, S. F. Fung, R. L. Kessel, S. H. Chen, J. L. Green, and T. E. Eastman, Geophys. Res. Lett. 25, 587 (1998).

${ }^{6}$ B. J. Anderson, R. E. Denton, G. Ho, D. C. Hamilton, and R. J. Strangeway, J. Geophys. Res. 101, 21527 (1996).

${ }^{7}$ S. Chandrasekhar, N. Kaufman, and K. Watson, Proc. R. Soc. London, Ser. A 245, 435 (1958).

${ }^{8}$ A. Barnes, Phys. Fluids 9, 1483 (1966).

${ }^{9}$ C. F. Kennel and H. E. Petschek, J. Geophys. Res. 71, 1 (1966).

${ }^{10}$ R. C. Davidson and Joan M. Ogden, Phys. Fluids 18, 1045 (1975).

${ }^{11}$ Gary R. Smith, Phys. Fluids 27, 1499 (1984).

${ }^{12}$ W. Manheimer and J. P. Boris, Comments Plasma Phys. Control. Fusion 3, 15 (1977).

${ }^{13}$ S. P. Gary, J. Wang, D. Winske, and S. A. Fuselier, J. Geophys. Res. 102, 27159 (1997).

${ }^{14}$ C. P. Price, D. W. Swift, and L.-C. Lee, J. Geophys. Res. 91, 101 (1986).

${ }^{15}$ S. P. Gary, S. A. Fuselier, and B. J. Anderson, J. Geophys. Res. 98, 1481 (1993).

${ }^{16}$ S. P. Gary, M. E. McKean, D. Winske, B. J. Anderson, R. E. Denton, and S. A. Fuselier, J. Geophys. Res. 99, 5903 (1994).

${ }^{17}$ T. A. Casper and Gary R. Smith, Phys. Rev. Lett. 48, 1015 (1982).

${ }^{18}$ R. Katsumata, M. Ichimura, M. Inutake, H. Hojo, A. Mase, and T. Tamano, Phys. Plasmas 3, 4489 (1996).

${ }^{19}$ M. Ichimura et al., Phys. Rev. Lett. 70, 2734 (1993)

${ }^{20}$ S. A. Fuselier, B. J. Anderson, S. P. Gary, and R. E. Denton, J. Geophys. Res. 99, 14931 (1994).

${ }^{21}$ P. A. Keiter, E. E. Scime, S. P. Gary, M. M. Balkey, R. F. Boivin, and J. L. Kline, Phys. Plasmas 7, 779 (2000).

${ }^{22}$ P. A. Keiter, E. E. Scime, and M. M. Balkey, Phys. Plasmas 4, 2741 (1997).

${ }^{23}$ V. E. Golant, A. P. Zhilinsky, and I. E. Sakharov, Fundamentals of Plasma Physics (Wiley, New York, 1977), p. 51.

${ }^{24}$ J. Gilland, R. Bruen, and N. Hershkowitz, Plasma Sources Sci. Technol. 7, 416 (1998).

${ }^{25}$ D. H. Hill, S. Fornaca, and M. G. Wickham, Rev. Sci. Instrum. 54, 309 (1983).

${ }^{26}$ E. E. Scime, P. A. Keiter, M. W. Zintl, M. M. Balkey, J. L. Kline, and M. E. Koepke, Plasma Sources Sci. Technol. 7, 186 (1998).

${ }^{27}$ J. L. Kline, E. E. Scime, P. A. Keiter, M. M. Balkey, and R. F. Boivin, Phys. Plasmas 6, 4767 (1999).

${ }^{28}$ P. A. Keiter, Ph.D. thesis, West Virginia University, 1999

${ }^{29}$ R. McWilliams and R. Koslover, Phys. Rev. Lett. 58, 37 (1987).

${ }^{30}$ M. Zintl and R. McWilliams, Rev. Sci. Instrum. 65, 2574 (1994).

${ }^{31}$ See for example, G. Ganguli, M. J. Keskinen, H. Remero, R. Heelis, T. Moore, and C. Pollock, J. Geophys. Res. 99, 8873 (1994). 
${ }^{32}$ J. D. Huba, NRL Plasma Formulary (Naval Research Laboratory, Washington, D.C., 1994), p. 33.

${ }^{33}$ A. V. Phelps, J. Phys. Chem. Ref. Data 20, 557 (1991).

${ }^{34}$ J. M. Beall, Y. C. Kim, and E. J. Power, J. Appl. Phys. 53, 3993 (1982).

${ }^{35}$ R. M. Kulsrud and C. S. Shen, Phys. Fluids 9, 177 (1966).
${ }^{36}$ R. K. Varma and D. Bhadra, Phys. Fluids 7, 1082 (1964).

${ }^{37}$ C. F. Kennel and H. E. Petschek, J. Geophys. Res. 71, 1 (1966).

${ }^{38}$ F. V. Coroniti, Space Sci. Rev. 42, 399 (1985).

${ }^{39}$ E. E. Scime, S. P. Gary, J. L. Phillips, and W. C. Feldman, J. Geophys. Res. 99, 23391 (1994). 